\title{
Information Measures of Ranked Set Samples In Farlie-Gumbel-Morgenstern Family
}

\author{
Saeid Tahmasebi ${ }^{1 *}$ and Ali Akbar Jafari ${ }^{2}$ \\ ${ }^{1}$ Department of Statistics, Persian Gulf University \\ ${ }^{2}$ Department of Statistics, Yazd University
}

\begin{abstract}
Ranked set sampling and some of its variants have been applied successfully in different areas of applications such as industrial statistics, economics, environmental and ecological studies, biostatistics, and statistical genetics. Ranked set sampling is a sampling method that more efficient than simple random sampling. Also, it is well known that Fisher information of a ranked set sample (RSS) is larger than Fisher information of a simple random sample (SRS) of the same size about the unknown parameter of the underlying distribution in parametric inference. In this paper, we consider the Farlie-Gumbel-Morgenstern (FGM) family and study the information measures such as Shannon's entropy, Rényi entropy, mutual information, and Kullback-Leibler (KL) information of RSS data. Also, we investigate their properties and compare them with a SRS data.
\end{abstract}

Key words: Concomitants of order statistics, Farlie-Gumbel-Morgenstern (FGM) family, Rényi entropy, Shannon entropy, Ranked set sampling.

\section{Introduction}

McIntyre (1952) first proposed ranked set sampling for estimating the mean pasture yields and indicated that ranked set sampling is a more efficient sampling method than simple random sampling method for estimating the population mean. The ranked set sampling technique is a sample selection procedure and composed of two stages. At the first stage of sample selection, $n$ simple random samples of size $n$ are drawn from an infinite population and each sample is called a set. Then, each of units are ranked from the smallest to the largest according to variable of interest, say $X$, in each set. Ranking of the units is done with a low-level measurement such as using previous experiences, visual measurement or using a concomitant variable. At the second stage, the first unit from the first set, the second unit from the second set and going on like this $n$th unit from the $n$th set are taken and measured according to the variable $X$ with a high level of measurement satisfying the desired sensitivity. The obtained sample is called a ranked set sample (RSS). Ranked set sampling and some of its variants have been applied successfully in different

\footnotetext{
${ }^{*}$ Corresponding author.
} 
areas of applications such as industrial statistics, environmental and ecological studies, biostatistics and statistical genetics. We assume that $\left(X_{S R S}, Y_{S R S}\right)=\left\{\left(X_{i}, Y_{i}\right), i=1,2,3, \cdots, n\right\}$ denotes a simple random sample (SRS) of size $n \geq 1$ from Farlie-Gumbel-Morgenstern (FGM) family with probability density function (pdf) given by

$$
f_{X, Y}(x, y)=f_{X}(x) f_{Y}(y)\left[1+\alpha\left(2 F_{X}(x)-1\right)\left(2 F_{Y}(y)-1\right)\right],
$$

where $-1 \leq \alpha \leq 1$, and $f_{X}(x), f_{Y}(y)$, and $F_{X}(x), F_{Y}(y)$ are marginal pdf and cumulative distribution function (cdf) of $X$ and $Y$, respectively. Also, the copula model of FGM family is defined by Nelson (1999) as follows:

$$
c(u, v)=[1+\alpha(1-2 u)(1-2 v)], 0 \leq u, v \leq 1 .
$$

Stokes (1977) applied ranked set sampling for bivariate random variable $(X, Y)$, where $X$ is the variable of interest and $Y$ is a concomitant variable that is not of direct interest but is relatively easy to measure. The procedure of ranked set sampling described by Stokes (1977) for a bivariate random variable is as follows:

Step 1. Randomly select $n$ independent bivariate samples, each of size $n$.

Step 2. Rank the units within each sample with respect to a variable of interest $X$ together with the $Y$ variate associated.

Step 3. In the $r$ th sample of size $n$, select the unit $\left(X_{(r) r}, Y_{[r] r}\right), r=1,2, \ldots, n$.

Let $X_{(r) r}$ be the measured observation on the variable $X$ in the $r$ th unit of the RSS and let $Y_{[r] r}$ be the corresponding measurement made on the study variable $Y$ of the same unit, $r=$ $1,2,3, \cdots, n$. Then clearly $Y_{[r] r}$ is the concomitant of $r$ th order statistic arising from the $r$ th sample. Also assume that $\left(X_{R S S}, Y_{[R S S]}\right)=\left\{\left(X_{(r) r}, Y_{[r] r}\right), r=1,2,3, \cdots, n\right\}$ denotes a RSS of size $n$ from bivariate distribution where the joint pdf of $\left(X_{(r) r}, Y_{[r] r}\right)$ is given by

$$
f_{X_{(r) r}, Y_{[r] r}}(x, y)=\frac{n !}{(r-1) !(n-r) !} f_{X, Y}(x, y)\left[F_{X}(x)\right]^{r-1}\left[1-F_{X}(x)\right]^{n-r}, \quad 1 \leq r \leq n .
$$

From Scaria and Nair (1999), the pdf of $Y_{[r] r}$ in FGM family is given by

$$
f_{Y_{[r] r}}(y)=\int_{-\infty}^{+\infty} f_{Y \mid X}(y \mid x) f_{X_{(r) r}}(x) d x=f_{Y}(y)\left[1+\frac{n-2 r+1}{n+1} \alpha\left(1-2 F_{Y}(y)\right)\right]
$$

Note that $f_{Y}(y)=\frac{1}{n} \sum_{r=1}^{n} f_{Y_{[r] r}}(y)$ and the pdf of $X_{(r) r}$ is

$$
f_{X_{(r) r}}(x)=\frac{n !}{(r-1) !(n-r) !} f_{X}(x)\left[F_{X}(x)\right]^{r-1}\left[1-F_{X}(x)\right]^{n-r}
$$

For perfect ranking situation, $X_{R S S}$ consists of independent order statistics from $F$. When ranking is imperfect, the rth judgment order statistic, will no longer be the rth order statistic. 
When ranking is imperfect, we use $X_{R S S}^{*}=\left\{\left(X_{(r) r}^{*}, r=1,2,3, \cdots, n\right\}\right.$ to denote an imperfect RSS of size $n$ from $f_{X}(x)$. Therefore, the pdf of $X_{(r) r}^{*}$ is shown by

$$
f_{X_{(r) r}^{*}}\left(x^{*}\right)=\sum_{r^{\prime}=1}^{n} p_{r, r^{\prime}} f_{X_{(r) r^{\prime}}}(x),
$$

where $p_{r, r^{\prime}}=\mathbb{P}\left(X_{(r)}=X_{\left(r^{\prime}\right) r^{\prime}}\right)$, denotes the probability with which the $r$ th order statistic is judged as having rank $r^{\prime}$ with $\sum_{r^{\prime}=1}^{n} p_{r, r^{\prime}}=\sum_{r=1}^{n} p_{r, r^{\prime}}=1$.

Also, the copula model for the joint pdf of $\left(X_{(r) r}, Y_{[r] r}\right)$ in FGM family is given by

$$
c_{r}(u, v)=\frac{n !}{(r-1) !(n-r) !}[1+\alpha(1-2 u)(1-2 v)] u^{r-1}(1-u)^{n-r} .
$$

Chen (2000) and Barabesi and El-Sharaawi (2001) studied the Fisher information contained in the usual RSS data for multi parameter family of distributions and showed that it is always larger than the Fisher information of the SRS data. Park and Lim (2012) studied the effect of imperfect ranking on the amount of Fisher information in ranked set samples. Frey (2013) showed that the Fisher information in an imperfect RSS may be higher than the Fisher information in a perfect RSS. In FGM family, we study the information measures such as Shannon's entropy, Rényi entropy, mutual information, and Kullback-Leibler (KL) information of RSS data. Also, we compare information measures of RSS data with SRS data. These measures are used in various contexts of ordered data such as order statistics by Wong and Chen (1990) and Park (1995), Ebrahimi et al. (2004), Bratpour et al.(2007a, b), record values by Raqab and Awad (2000, 2001), Zahedi and Shakil (2006), Ahmadi and Fashandi (2008), Madadi and Tata (2011), and in testing hypothesis by Park (2005), Balakrishnan et al. (2007) and Habibi Rad et al. (2011), and censored data by Abo-Eleneen(2011). Abo-Eleneen (2001) and Abo-Eleneen and Nagaraja (2002a) studied Fisher information in pairs and collections of order statistics and their concomitants from bivariate samples. Tahmasebi and Behboodian (2012) obtained some results of information measures for concomitants of order statistics in FGM family. Recently, Fashandi and Ahmadi (2012) studied characterizations of symmetric distributions based on Rényi entropy of concomitants.

The organization of this article is as follows: In Section 2, we obtain the Shannon entropy for RSS in FGM family and illustrate for three special cases. In Section 3, the Rényi entropy for RSS in FGM family is presented. Mutual information for RSS is given in Section 4. Kullback-Leibler information for RSS is presented in Section 5.

\section{Shannon entropy of RSS in FGM family}

Shannon entropy is a mathematical measure of information which measures the average reduction of uncertainty for a continuous random variable X, and defined by Shannon (1948) as

$$
H(X)=-\int_{-\infty}^{+\infty} f_{X}(x) \log f_{X}(x) d x=-\int_{0}^{1} \log f_{X}\left(F_{X}^{-1}(u)\right) d u
$$

where $f_{X}(x)$ is the pdf of $X$.

Entropy has also been used in various branches of statistics and related fields, and has become an integral part of probability and statistics (Soofi, 2000). Harris (1982) regarded Shannon 
entropy as a descriptive quantity of the corresponding probability distribution, and Nayak (1985) justified its utility as a descriptive measure. We refer the reader to Cover and Thomas (1991) as reference therein for more details.

The Shannon entropy of $\left(X_{S R S}, Y_{S R S}\right)$ is given by

$$
H\left(X_{S R S}, Y_{S R S}\right)=-\sum_{i=1}^{n} \iint f_{X_{i}, Y_{i}}\left(x_{i}, y_{i}\right) \log f_{X_{i}, Y_{i}}\left(x_{i}, y_{i}\right) d x_{i} d y_{i}=n H\left(X_{1}, Y_{1}\right) .
$$

Also, under the perfect ranking assumption in bivariate distribution, we have

$$
H\left(X_{R S S}, Y_{[R S S]}\right)=-\sum_{r=1}^{n} \iint f_{(r) r,[r] r}(x, y) \log f_{(r) r,[r] r}(x, y) d x d y=\sum_{r=1}^{n} H\left(X_{(r) r}, Y_{[r] r}\right) .
$$

Lemma 2.1. 1A general expression for $H\left(X_{(r) r}, Y_{[r] r}\right)$ is given

$$
H\left(X_{(r) r}, Y_{[r] r}\right)=-\log a_{r}+a_{r}\left[(r-1) Z_{n}(r)+(n-r) Z_{n}(n-r+1)\right]+Q_{n}(r),
$$

where $a_{r}=\frac{n !}{(r-1) !(n-r) !}=n\left(\begin{array}{c}n-1 \\ r-1\end{array}\right)$ and

$$
Z_{n}(r)=\sum_{m=0}^{n-r}\left(\begin{array}{c}
n-r \\
m
\end{array}\right) \frac{(-1)^{m}}{(m+r)^{2}} \quad, \quad Q_{n}(r)=E_{f_{(r) r,[r] r}}[-\log f(X, Y)] .
$$

Proof. From (1.3) and (2.1), we have

$$
\begin{aligned}
H\left(X_{(r) r}, Y_{[r] r}\right) & =-E_{f_{(r) r,[r] r}}\left[\log f_{X_{(r) r}, Y_{[r] r}}(X, Y)\right] \\
& =-E_{f_{(r) r,[r] r}}\left[\log a_{r}+(r-1) \log F(X)+(n-r) \log (1-F(X))+\log f(X, Y)\right] \\
& =-\log a_{r}+a_{r}\left[(r-1) A_{1}+(n-r) A_{2}\right]+Q_{n}(r),
\end{aligned}
$$

where

$$
Q_{n}(r)=\int_{-\infty}^{+\infty} \int_{-\infty}^{+\infty}\left(-\log f_{X, Y}(x, y)\right) a_{r} f_{X, Y}(x, y)\left[F_{X}(x)\right]^{r-1}\left[1-F_{X}(x)\right]^{n-r} d x d y,
$$

and

$$
\begin{aligned}
A_{1} & =\int_{-\infty}^{+\infty} \int_{-\infty}^{+\infty}\left(-\log F_{X}(x)\right)\left[F_{X}(x)\right]^{r-1}\left[1-F_{X}(x)\right]^{n-r} f_{X, Y}(x, y) d y d x \\
& =\int_{-\infty}^{+\infty}\left(-\log F_{X}(x)\right)\left[F_{X}(x)\right]^{r-1}\left[1-F_{X}(x)\right]^{n-r} f_{X}(x) d x \\
& =\int_{0}^{1}(-\log u) u^{r-1}(1-u)^{n-r} d u=\int_{0}^{1}(-\log u) u^{r-1} \sum_{m=0}^{n-r}\left(\begin{array}{c}
n-r \\
m
\end{array}\right)(-u)^{m} d u \\
& =\sum_{m=0}^{n-r}\left(\begin{array}{c}
n-r \\
m
\end{array}\right) \frac{(-1)^{m}}{(m+r)^{2}}=Z_{n}(r),
\end{aligned}
$$


and similarly,

$$
\begin{aligned}
A_{2} & =\int_{-\infty}^{+\infty} \int_{-\infty}^{+\infty}\left[-\log \left(1-F_{X}(x)\right)\right]\left[F_{X}(x)\right]^{r-1}\left[1-F_{X}(x)\right]^{n-r} f_{X, Y}(x, y) d y d x \\
& =\int_{-\infty}^{+\infty}\left[-\log \left(1-F_{X}(x)\right)\right]\left[F_{X}(x)\right]^{r-1}\left[1-F_{X}(x)\right]^{n-r} f_{X}(x) d x \\
& =\int_{0}^{1}[-\log (1-u)] u^{r-1}(1-u)^{n-r} d u=\int_{0}^{1}(-\log (1-u)) u^{r-1} \sum_{m=0}^{n-r}\left(\begin{array}{c}
n-r \\
m
\end{array}\right)(-u)^{m} d u \\
& =\sum_{m=0}^{r-1}\left(\begin{array}{c}
r-1 \\
m
\end{array}\right) \frac{(-1)^{m}}{(m+n-r+1)^{2}}=Z_{n}(n-r+1) .
\end{aligned}
$$

So the proof is completed by substitution.

Remark 2.1. 2 Suppose $(X, Y)$ have bivariate standard normal distribution with pdf

$$
\varphi_{2}(x, y ; \rho)=\frac{1}{2 \pi \sqrt{1-\rho^{2}}} \exp \left\{\frac{-\left(x^{2}-2 \rho x y+y^{2}\right)}{2\left(1-\rho^{2}\right)}\right\} .
$$

Then by using (2.3), we have

$$
\begin{aligned}
H\left(X_{(r) r}, Y_{[r] r}\right) & =-\log a_{r}+a_{r}\left[(r-1) Z_{n}(r)+(n-r) Z_{n}(n-r+1)\right] \\
& +\int_{-\infty}^{+\infty} \int_{-\infty}^{+\infty} a_{r}\left[-\log \varphi_{2}(x, y ; \rho)\right] \varphi_{2}(x, y ; \rho)[\Phi(x)]^{r-1}[1-\Phi(x)]^{n-r} d x d y \\
& =-\log a_{r}+a_{r}\left[(r-1) Z_{n}(r)+(n-r) Z_{n}(n-r+1)\right]+0.5+\log \left(2 \pi \sqrt{1-\rho^{2}}\right) \\
& +\frac{a_{r}}{2} \sum_{m=0}^{n-r}\left(\begin{array}{c}
n-r \\
m
\end{array}\right)(-1)^{m} \int_{-\infty}^{+\infty} x^{2}[\Phi(x)]^{r+m-1} \phi(x) d x,
\end{aligned}
$$

where $\phi$ and $\Phi$ denote, as usual, the pdf and cdf of the standard normal distribution, respectively.

The copula density of bivariate normal with parameter $\rho$ is obtained by Meyer (2009) as follows:

$$
\begin{aligned}
c(u, v ; \rho) & =\frac{\partial^{2}}{\partial u \partial v} \Phi_{2}\left(\Phi^{-1}(u), \Phi^{-1}(v) ; \rho\right)=\frac{\varphi_{2}\left(\Phi^{-1}(u), \Phi^{-1}(v) ; \rho\right)}{\varphi\left(\Phi^{-1}(u)\right) \varphi\left(\Phi^{-1}(v)\right)} \\
& =\frac{1}{\sqrt{1-\rho^{2}}} \exp \left\{\frac{2 \rho \Phi^{-1}(u) \Phi^{-1}(v)-\rho^{2}\left[\left(\Phi^{-1}(u)\right)^{2}+\left(\Phi^{-1}(v)\right)^{2}\right]}{2\left(1-\rho^{2}\right)}\right\}
\end{aligned}
$$

where $\Phi_{2}(h, k ; \rho)=\int_{-\infty}^{h} \int_{-\infty}^{k} \varphi_{2}(x, y ; \rho) d y d x$.

Also, the copula model for the joint pdf of $\left(X_{(r) r}, Y_{[r] r}\right)$ in bivariate standard normal distribution is given by

$$
c_{r}(u, v)=a_{r} c(u, v ; \rho) u^{r-1}(1-u)^{n-r} .
$$


Remark 2.2.3 We assume that $\left(X_{R S S}, Y_{[R S S]}\right)=\left\{\left(X_{(r) r}, Y_{[r] r}\right), r=1,2,3, \cdots, n\right\}$ denotes a RSS of sizenfrom the copula model of bivariate standard normal distribution, then by using (2.3) we have

$$
\begin{aligned}
H\left(X_{R S S}, Y_{[R S S]}\right) & =\sum_{r=1}^{n} H\left(X_{(r) r}, Y_{[r] r}\right)=\sum_{r=1}^{n}\left\{-\log a_{r}+a_{r}\left[(r-1) Z_{n}(r)+(n-r) Z_{n}(n-r+1)\right]\right. \\
& \left.+\int_{0}^{1} \int_{0}^{1} a_{r}[-\log c(u, v ; \rho)] c(u, v ; \rho)(u)^{r-1}(1-u)^{n-r} d u d v\right\} \\
& =\frac{n}{2} \log \left(1-\rho^{2}\right)+\sum_{r=1}^{n}\left\{-\log a_{r}+a_{r}\left[(r-1) Z_{n}(r)+(n-r) Z_{n}(n-r+1)\right]\right. \\
& \left.-\frac{1}{2\left(1-\rho^{2}\right)} \int_{0}^{1} \int_{0}^{1}\left[2 \rho \Phi^{-1}(u) \Phi^{-1}(v)-\rho^{2}\left[\left(\Phi^{-1}(u)\right)^{2}+\left(\Phi^{-1}(v)\right)^{2}\right]\right] c_{r}(u, v) d u d v\right\} .
\end{aligned}
$$

Under the perfect ranking assumption, it is easy to see that

$$
H\left(X_{R S S}\right)=-\sum_{r=1}^{n} \int f_{(r) r}(x) \log f_{(r) r}(x) d x=\sum_{r=1}^{n} H\left(X_{(r) r}\right),
$$

where $H\left(X_{(r) r}\right)$ is the entropy of the $r$ th order statistic in a sample of size $n$. Ebrahimi et al. (2004) presented a general expression for $H\left(X_{(r) r}\right)$ as

$$
H\left(X_{(r) r}\right)=H\left(U_{(r) r}\right)-E\left[\log \left[f\left(F^{-1}\left(W_{r}\right)\right)\right]\right],
$$

where $H\left(U_{(r) r}\right)$ is the entropy of $r$ th order statistic from a random sample of size $n$ from a uniform $(0,1)$ distribution and $W_{r}$ has the beta distribution with parameters $r$ and $n-r+1$. Jafari Jozani and Ahmadi (2014) obtained an expression for $H\left(X_{R S S}\right)$ which is given by

$$
H\left(X_{R S S}\right)=H\left(X_{S R S}\right)+k(n)
$$

where

$$
\begin{aligned}
k(n)= & \sum_{r=1}^{n} H\left(U_{(r) r}\right)=\sum_{r=1}^{n}[\log (B(r, n-r+1))-(r-1)(\psi(r)-\psi(n+1)) \\
& -(n-r)(\psi(n-r+1)-\psi(n+1))] \\
= & 2 \sum_{j=1}^{n-1}(n-2 j) \log (j)-n \log (n)-2 \sum_{r=1}^{n}(r-1) \psi(r)+n(n-1) \psi(n+1),
\end{aligned}
$$

where $B(a, b)$ is the beta function, $\psi(n)=\frac{d \log \Gamma(n)}{d n}$ is the digamma function and $\psi(n+1)=$ $\psi(n)+\frac{1}{n}$. Also, under the perfect ranking assumption, it is easy to see that

$$
H\left(Y_{[R S S]}\right)=-\sum_{r=1}^{n} \int g_{[r] r}(y) \log g_{[r] r}(y) d y=\sum_{r=1}^{n} H\left(Y_{[r] r}\right),
$$


where $H\left(Y_{[r] r}\right)$ is the Shannon entropy for concomitant of $r$ th order statistic in FGM family. Tahmasebi and Behboodian (2012) presented a general expression for $H\left(Y_{[r] r}\right)$ as

$$
\begin{aligned}
H\left(Y_{[r] r}\right) & =I_{\alpha, n}(r)-E_{g_{[r] r}}(\log f(Y)) \\
& =I_{\alpha, n}(r)+H(Y)\left(1+\left(\frac{n-2 r+1}{n+1}\right) \alpha\right)+2 \alpha\left(\frac{n-2 r+1}{n+1}\right) \phi(f),
\end{aligned}
$$

where

$$
\begin{aligned}
I_{\alpha, n}(r)= & \frac{n+1}{8 \alpha(n-2 r+1)}\left\{\left(1-\left(\frac{n-2 r+1}{n+1}\right) \alpha\right)^{2}\left[2 \log \left(1-\left(\frac{n-2 r+1}{n+1}\right) \alpha\right)-1\right]\right. \\
& \left.-\left(1+\left(\frac{n-2 r+1}{n+1}\right) \alpha\right)^{2}\left[2 \log \left(1+\left(\frac{n-2 r+1}{n+1}\right) \alpha\right)-1\right]\right\},
\end{aligned}
$$

and

$$
\phi(f)=\int_{0}^{1} u \log f_{Y}\left(F_{Y}^{-1}(u)\right) d u
$$

For developing our results here, we shall use the following lemmas, established recently by Jafari Jozani and Ahmadi (2013).

Lemma 2.2. $4 H\left(X_{R S S}\right) \leq H\left(X_{S R S}\right)$ for all set size $n \in \mathbb{N}$ and the equality holds when $n=1$.

Lemma 2.3.5 $H\left(X_{R S S}\right) \leq H\left(X_{R S S}^{*}\right)$ for all set size $n \in \mathbb{N}$ and the equality happens when the ranking is perfect.

Lemma 2.4. $6 H\left(X_{R S S}^{*}\right) \leq H\left(X_{S R S}\right)$ for all set size $n \in \mathbb{N}$ and the equality holds when the ranking is done randomly and $p_{r, r^{\prime}}=\mathbb{P}\left(X_{(r)}=X_{\left(r^{\prime}\right) r^{\prime}}\right)=\frac{1}{n}$ for all $r, r^{\prime} \in\{1,2, \ldots, n\}$.

Also, an ordering relationship among the Shannon entropies of $X_{R S S}^{*}, X_{R S S}$ and $X_{S R S}$ is obtained by Jafari Jozani and Ahmadi (2014) as

$$
H\left(X_{R S S}\right) \leq H\left(X_{R S S}^{*}\right) \leq H\left(X_{S R S}\right) .
$$

Lemma 2.5. 7 Let $\left(X_{R S S}, Y_{[R S S]}\right)=\left\{\left(X_{(r) r}, Y_{[r] r}\right), r=1,2,3, \cdots, n\right\}$ be a ranked set sample of size $n$ from FGM family, then

$$
H\left(X_{R S S}, Y_{[R S S]}\right)=H\left(X_{R S S}\right)+H\left(Y_{S R S}\right)+H\left(\tilde{X}_{S R S}, \tilde{Y}_{S R S}\right),
$$

where $H\left(Y_{S R S}\right)=-n \int f_{Y}(y) \log f_{Y}(y) d y$ and $H\left(\tilde{X}_{S R S}, \tilde{Y}_{S R S}\right)$ is the Shannon entropy for $\left.X_{S R S}, Y_{S R S}\right)$ in the copula model of FGM family. 
Proof.

$$
\begin{aligned}
H\left(X_{R S S}, Y_{[R S S]}\right)= & H\left(X_{R S S}\right)+H\left(Y_{[R S S]} \mid X_{R S S}\right) \\
= & H\left(X_{R S S}\right)-\sum_{r=1}^{n} \iint \log f_{Y_{[r] r} \mid X_{(r) r}}(y \mid x) f_{(r) r,[r] r}(x, y) d x d y \\
= & H\left(X_{R S S}\right)-\sum_{r=1}^{n} \iint \log f_{Y \mid X}(y \mid x) f_{(r) r,[r] r}(x, y) d x d y \\
= & H\left(X_{R S S}\right)-\sum_{r=1}^{n} \iint \log \left\{f _ { Y } ( y ) \left[1+\alpha\left(2 F_{X}(x)-1\right)\right.\right. \\
= & H\left(X_{R S S}\right)-\sum_{r=1}^{n} \iint \log f_{Y}(y) f_{(r) r,[r] r}(x, y) d x d y \\
& \left.\left.\left.-\sum_{r=1}^{n} \iint_{Y}(y)-1\right)\right]\right\} f_{(r) r,[r] r}(x, y) d x d y \\
= & H\left(X_{R S S}\right)-n \int f_{Y}(y) \log f_{Y}(y) d y \\
& -n \int_{0}^{1} \int_{0}^{1} \log [1+\alpha(2 u-1)(2 v-1)][1+\alpha(2 u-1)(2 v-1)] d u d v \\
= & H\left(X_{R S S}\right)+H\left(Y_{S R S}\right)+H\left(\tilde{X}_{S R S}, \tilde{Y}_{S R S}\right) .
\end{aligned}
$$

In the following lemma we observe that the Shannon entropy of $Y_{[R S S]}$ is less than the Shannon entropy of $Y_{S R S}$ in FGM family.

Lemma 2.6. 8H( $\left.Y_{[R S S]}\right) \leq H\left(Y_{S R S}\right)$ for all set sizen $\in \mathbb{N}$ and the equality holds when $n=1$ or $\alpha=0$.

Proof. By using (11) and (12), we have

$$
\begin{aligned}
H\left(Y_{[R S S]}\right) & =\sum_{r=1}^{n} H\left(Y_{[r] r}\right)=\sum_{r=1}^{n} I_{\alpha, n}(r)-\int \sum_{r=1}^{n} g_{[r] r}(y) \log f_{Y}(y) d y \\
& =\sum_{r=1}^{n} I_{\alpha, n}(r)-n \int f_{Y}(y) \log f_{Y}(y) d y \\
& =w_{\alpha}(n)+H\left(Y_{S R S}\right),
\end{aligned}
$$

where $w_{\alpha}(n)=\sum_{r=1}^{n} I_{\alpha, n}(r)$.

Note that $H\left(Y_{[R S S]}\right) \leq H\left(Y_{S R S}\right)$ since $w_{\alpha}(n) \leq 0$ (see Example 1, Tahmasebi and Behboodian, 2012). Also, $H\left(Y_{[R S S]}\right)-H\left(Y_{S R S}\right)=w_{\alpha}(n)$ is distribution-free (doesn't depend on the parent distribution). 
Remark 2.3.9 Another expressions for $H\left(Y_{[R S S]}\right), H\left(X_{R S S}\right)$ and $H\left(X_{R S S}, Y_{[R S S]}\right)$ in FGM family are given by

$$
\begin{aligned}
& H\left(Y_{[R S S]}\right)=H\left(\tilde{Y}_{[R S S]}\right)+H\left(Y_{S R S}\right), \\
& \begin{array}{c}
H\left(X_{R S S}\right)=H\left(\tilde{X}_{R S S}\right)+H\left(X_{S R S}\right), \\
H\left(X_{R S S}, Y_{[R S S]}\right)=H\left(\tilde{X}_{R S S}\right)+H\left(X_{S R S}\right)+H\left(Y_{S R S}\right)+H\left(\tilde{X}_{S R S}, \tilde{Y}_{S R S}\right) \\
\quad=H\left(\tilde{X}_{R S S}, \tilde{Y}_{[R S S]}\right)+H\left(X_{S R S}\right)+H\left(Y_{S R S}\right)
\end{array}
\end{aligned}
$$

where $H\left(\tilde{X}_{R S S}\right)=k(n)$ and $H\left(\tilde{Y}_{[R S S]}\right)=w_{\alpha}(n)$ are the entropies of $X_{R S S}$ and $Y_{[R S S]}$ in the copula model of FGM family. Note that

$$
H\left(\tilde{X}_{R S S}, \tilde{Y}_{[R S S]}\right)=H\left(\tilde{X}_{R S S}\right)+H\left(\tilde{X}_{S R S}, \tilde{Y}_{S R S}\right),
$$

is the joint entropy for $\left(X_{R S S}, Y_{[R S S]}\right)$ in the copula model of FGM family.

Example 2.1. 10Let $\left(X_{i}, Y_{i}\right), i=1,2, \cdots, n$ be a random sample from (1.1). Then, the density function of $Y_{[r] r}$ is

$$
\mathrm{g}_{[r] r}(y)=1+\alpha\left(\frac{n-2 r+1}{n+1}\right)(1-2 y) .
$$

Now, by using (2.6) and (2.7), we can easily show that $H\left(\tilde{Y}_{[R S S]}\right), H\left(\tilde{X}_{R S S}\right)$ and $H\left(\tilde{X}_{R S S}, \tilde{Y}_{[R S S]}\right)$ for the copula model of FGM family have the following properties

$$
\begin{aligned}
& \text { (i) } H\left(\tilde{Y}_{[R S S]}\right)=w_{\alpha}(n)=w_{-\alpha}(n)=\sum_{r=1}^{n} I_{\alpha, n}(n-r+1), \\
& \text { (ii) } H\left(\tilde{X}_{R S S}\right)<H\left(\tilde{Y}_{[R S S]}\right), \quad 1 \leq r \leq n, \\
& \text { (iii) } H\left(\tilde{X}_{R S S}\right)=k(n)=\sum_{r=1}^{n} H\left(U_{(n-r+1) n-r+1}\right)<0, \quad 1 \leq r \leq n, \\
& \text { (iv) }-0.19 n<H\left(\tilde{Y}_{[R S S]}\right)<0, \quad n \geq 2, \\
& \text { (v) } H\left(\tilde{X}_{R S S}, \tilde{Y}_{[R S S]}\right)=H\left(\tilde{X}_{R S S}\right)+H\left(\tilde{X}_{S R S}, \tilde{Y}_{S R S}\right)<0, \\
& \text { (vi) } H\left(\tilde{X}_{R S S}, \tilde{Y}_{[R S S]}\right)<H\left(\tilde{X}_{S R S}, \tilde{Y}_{S R S}\right), \\
& \text { (vii) } H\left(\tilde{X}_{R S S}, \tilde{Y}_{[R S S]}\right)<H\left(\tilde{Y}_{[R S S]}\right), \\
& \text { (viii) } H\left(\tilde{X}_{R S S}, \tilde{Y}_{[R S S]}\right)<H\left(\tilde{X}_{R S S}\right) .
\end{aligned}
$$

Table 1 provides values of $H\left(\tilde{Y}_{[R S S]}\right)$ and $H\left(\tilde{X}_{R S S}\right)$ for $n=3(2) 9$ and $\alpha= \pm .25(.25) \pm 1$. For fixed $n, H\left(\tilde{Y}_{[R S S]}\right)$ is increasing (decreasing) in $\alpha$ for $-1 \leq \alpha<0(0<\alpha \leq 1)$. As Table 1 shows, $H\left(\tilde{X}_{R S S}\right)$ is smaller than $H\left(\tilde{Y}_{[R S S]}\right)$ and $H\left(\tilde{X}_{R S S}\right)$ is decreasing in $n$. Also, for fixed $\alpha$, $H\left(\tilde{Y}_{[R S S]}\right)$ is decreasing in $n$. Table 1 also provides values of $H\left(\tilde{X}_{R S S}, \tilde{Y}_{[R S S]}\right)$ for $n=5(5) 20$ and $\alpha= \pm .25(.25) \pm 1$. For fixed $n, H\left(\tilde{X}_{R S S}, \tilde{Y}_{[R S S]}\right)$ is increasing (decreasing) in $\alpha$ for $-1 \leq$ $\alpha<0(0<\alpha \leq 1)$. As Table 1 shows, $H\left(\tilde{X}_{R S S}, \tilde{Y}_{[R S S]}\right)$ is smaller than $H\left(\tilde{X}_{S R S}, \tilde{Y}_{S R S}\right)$ and $H\left(\tilde{X}_{S R S}, \tilde{Y}_{S R S}\right)$ is decreasing in $n$. Also, for fixed $\alpha, H\left(\tilde{X}_{R S S}, \tilde{Y}_{[R S S]}\right)$ is decreasing in $n$. 
Table 1. $H\left(\tilde{Y}_{[R S S]}\right), H\left(\tilde{X}_{R S S}\right), H\left(\tilde{X}_{S R S}, \tilde{Y}_{S R S}\right)$ and $H\left(\tilde{X}_{R S S}, \tilde{Y}_{[R S S]}\right)$ for the copula model of FGM family.

\begin{tabular}{|c|c|c|c|c|c|c|}
\hline & $\alpha$ & \pm 0.25 & \pm 0.5 & \pm 0.75 & \pm 1 & \\
\hline & \multicolumn{5}{|c|}{$H\left(\tilde{Y}_{[R S S]}\right)=w_{\alpha}(n)$} & $H\left(\tilde{X}_{R S S}\right)=k(n)$ \\
\hline$n$ & 3 & -0.0052 & -0.0208 & -0.0474 & -0.0854 & -0.9888 \\
& 5 & -0.0114 & -0.0458 & -0.1062 & -0.1930 & -2.6110 \\
& 7 & -0.0180 & -0.0734 & -0.1680 & -0.3064 & -4.6162 \\
& 9 & -0.0246 & -0.1008 & -0.2312 & -0.4222 & -6.8970 \\
\hline & \multicolumn{6}{|c|}{$H\left(\tilde{X}_{S R S}, \tilde{Y}_{S R S}\right)$} \\
\hline$n$ & 5 & -0.0390 & -0.0590 & -0.1690 & -0.2890 & \\
& 10 & -0.0424 & -0.1562 & -0.3468 & -0.6293 & \\
& 15 & -0.0633 & -0.2338 & -0.5202 & -0.9435 & \\
& 20 & -0.0848 & -0.3124 & -0.6936 & -1.2586 & \\
\hline & \multicolumn{7}{|c|}{$H\left(\tilde{X}_{R S S}, \tilde{Y}_{[R S S]}\right)$} \\
\hline$n$ & 5 & -2.65 & -2.67 & -2.78 & -2.90 & \\
& 10 & -8.16 & -8.26 & -8.46 & -8.67 & \\
& 15 & -14.93 & -15.12 & -15.36 & -15.41 & \\
& 20 & -22.54 & -22.80 & -23.16 & -23.22 & \\
\hline
\end{tabular}

Example 2.2 11Let $\left(X_{i}, Y_{i}\right), i=1,2, \cdots, n$ be a random sample from Gumbel's bivariate exponential distribution with $c d f$

$$
F_{X, Y}(x, y)=\left(1-\exp \left(\frac{-x}{\theta_{1}}\right)\right)\left(1-\exp \left(\frac{-y}{\theta_{2}}\right)\right)\left[1+\alpha \exp \left(\frac{-x}{\theta_{1}}-\frac{y}{\theta_{2}}\right)\right]
$$

In this case, the pdf of $Y_{[r] r}$ is

$$
g_{[r] r}(y)=\frac{1}{\theta_{2}} \exp \left(\frac{-y}{\theta_{2}}\right)\left[1+\alpha\left(\frac{n-2 r+1}{n+1}\right)\left(2 \exp \left(\frac{-y}{\theta_{2}}\right)-1\right)\right]
$$

Also, the entropies of $X_{R S S}$ and $Y_{[R S S]}$ are given by

$$
\begin{gathered}
H\left(X_{R S S}\right)=k(n)+n \log \theta_{1}+n, \\
H\left(Y_{[R S S]}\right)=w_{\alpha}(n)+n \log \theta_{2}+n .
\end{gathered}
$$

Now, if $\theta_{1} \leq \theta_{2}$, then for fixed $\alpha$ and $n \geq 2$, we have

$$
H\left(X_{R S S}\right)-H\left(Y_{[R S S]}\right)=n \log \left(\frac{\theta_{1}}{\theta_{2}}\right)+k(n)-w_{\alpha}(n)<0 .
$$

Example 2.3. 12Let $\left(X_{i}, Y_{i}\right), i=1,2, \cdots$, nbe a random sample from standard Morgenstern type bivariate logistic distribution with $c d f$

$$
F(x, y)=(1+\exp (-x))^{-1}(1+\exp (-y))^{-1}\left\{1+\frac{\alpha e^{-x-y}}{\left(1+e^{-x}\right)\left(1+e^{-y}\right)}\right\}, \quad-\infty<x, y<+\infty
$$

The pdf of $Y_{[r] r}$ is

$$
g_{[r] r}(y)=e^{-y}\left(1+e^{-y}\right)^{-2}\left\{1+\left(\frac{n-2 r+1}{n+1}\right)\left(1-2\left(1+e^{-y}\right)^{-1}\right) \alpha\right\} .
$$

Computations show that

$$
H\left(Y_{[R S S]}\right)=w_{\alpha}(n)+2=w_{\alpha}(n)+H\left(X_{R S S}\right)-k(n) .
$$

Therefore, 


$$
H\left(X_{R S S}\right)<H\left(Y_{[R S S]}\right)
$$

\section{Rényi entropy of RSS in FGM family}

In information theory, the Rényi entropy is a generaliztion for the Shannon entropy. The Rényi entropy of order $\lambda$ is defined as

$$
H_{\lambda}(X)=\frac{1}{1-\lambda} \log \int_{-\infty}^{+\infty} f_{X}^{\lambda}(x) d x,
$$

where $\lambda>0, \lambda \neq 1$, and $H(X)=\lim _{\lambda \rightarrow 1} H_{\lambda}(X)=-\int_{-\infty}^{\infty} f_{X}(x) \log f_{X}(x) d x$ is the Shannon entropy if both integrals exist.

The Rényi entropy of $\left(X_{S R S}, Y_{S R S}\right)$ is given by

$$
H_{\lambda}\left(X_{S R S}, Y_{S R S}\right)=\sum_{i=1}^{n} \frac{1}{1-\lambda} \log \iint\left[f_{X_{i}, Y_{i}}\left(x_{i}, y_{i}\right)\right]^{\lambda} d x_{i} d y_{i}=n H_{\lambda}\left(X_{1}, Y_{1}\right) .
$$

Also, under the perfect ranking assumption in FGM family, we have

$$
H_{\lambda}\left(X_{R S S}, Y_{[R S S]}\right)=\sum_{r=1}^{n} \frac{1}{1-\lambda} \log \iint\left[f_{(r) r,[r] r}(x, y)\right]^{\lambda} d x d y=\sum_{r=1}^{n} H_{\lambda}\left(X_{(r) r}, Y_{[r] r}\right),
$$

and it is easy to see that

$$
H_{\lambda}\left(X_{R S S}\right)=\sum_{r=1}^{n} \frac{1}{1-\lambda} \log \int\left[f_{(r) r}(x)\right]^{\lambda} d x=\sum_{r=1}^{n} H_{\lambda}\left(X_{(r) r}\right),
$$

where $H_{\lambda}\left(X_{(r) r}\right)$ is the Rényi entropy of the $r$ th order statistic in a sample of size $n$. Baratpour et al. (2008) presented a general expression for $H\left(X_{(r) r}\right)$ as

$$
H_{\lambda}\left(X_{(r) r}\right)=H_{\lambda}\left(V_{(r) r}\right)-\frac{1}{\lambda-1} \log E_{h_{r}}\left[f^{\lambda-1}\left(F^{-1}\left(Z_{r}\right)\right)\right],
$$

where $H_{\lambda}\left(V_{(r) r}\right)$ is the Rényi entropy of the beta distribution with parameters $r$ and $n-r+1$ and $Z_{r} \sim h_{r}$ where $h_{r}$ is the beta pdf with parameters $\lambda(r-1)+1$ and $\lambda(n-r)+1$. Note that Abbasnejad and Arghami (2011) obtained an expression for $H_{\lambda}\left(V_{(r) r}\right)$ as

$$
H_{\lambda}\left(V_{(r) r}\right)=\frac{\lambda}{\lambda-1} \log B(r, n-r+1)-\frac{1}{\lambda-1} \log B(\lambda(r-1)+1, \lambda(n-r)+1) .
$$

Also, under the perfect ranking assumption, it is easy to see that

$$
H_{\lambda}\left(Y_{[R S S]}\right)=\sum_{r=1}^{n} \frac{1}{1-\lambda} \log \int\left[g_{[r] r}(y)\right]^{\lambda} d y=\sum_{r=1}^{n} H_{\lambda}\left(Y_{[r] r}\right),
$$

where $H_{\lambda}\left(Y_{[r] r}\right)$ is the Rényi entropy for concomitant of $r$ th order statistic in FGM family. A general expression for $H\left(Y_{[r] r}\right)$ is given by 
where $U \sim$ Uniform $(0,1)$.

$$
\begin{aligned}
H\left(Y_{[r] r}\right) & =\frac{1}{1-\lambda} \log \int_{0}^{1}\left[1+\alpha\left(\frac{n-2 r+1}{n+1}\right)(1-2 u)\right]^{\lambda}\left[f_{Y}\left(F_{Y}^{-1}(u)\right)\right]^{\lambda-1} d u \\
& =\frac{1}{1-\lambda} \log E_{U}\left\{\left[g_{[r] r}(U)\right]^{\lambda}\left[f_{Y}\left(F_{Y}^{-1}(U)\right)\right]^{\lambda-1}\right\},
\end{aligned}
$$

An ordering relationship among the Rényi entropies of $X_{R S S}^{*}, X_{R S S}$ and $X_{S R S}$ is obtained by Jafari Jozani and Ahmadi (2014) as

$$
H_{\lambda}\left(X_{R S S}\right) \leq H_{\lambda}\left(X_{R S S}^{*}\right) \leq H_{\lambda}\left(X_{S R S}\right) .
$$

Lemma 3.1. 13 Let $\left(X_{R S S}, Y_{[R S S]}\right)=\left\{\left(X_{(r) r}, Y_{[r] r}\right), r=1,2,3, \cdots, n\right\}$ be a ranked set sample of size $n$ from FGM family, then

$$
H_{\lambda}\left(X_{R S S}, Y_{[R S S]}\right)=H_{\lambda}\left(X_{R S S}\right)+n H_{\lambda}(Y \mid X) .
$$

Proof.

$$
\begin{aligned}
H_{\lambda}\left(X_{R S S}, Y_{[R S S]}\right) & =\sum_{r=1}^{n} \frac{1}{1-\lambda} \log \iint\left[f_{(r) r,[r] r}(x, y)\right]^{\lambda} d x d y \\
& =H_{\lambda}\left(X_{R S S}\right)+H_{\lambda}\left(Y_{[R S S]} \mid X_{R S S}\right) \\
& =H_{\lambda}\left(X_{R S S}\right)+\sum_{r=1}^{n} \frac{1}{1-\lambda} \log \int\left[f_{Y_{[r] r} \mid X_{(r) r}}(y \mid x)\right]^{\lambda} d y \\
& =H_{\lambda}\left(X_{R S S}\right)+\sum_{r=1}^{n} \frac{1}{1-\lambda} \log \int\left[f_{Y \mid X}(y \mid x)\right]^{\lambda} d y \\
& =H_{\lambda}\left(X_{R S S}\right)+n H_{\lambda}(Y \mid X) .
\end{aligned}
$$

Analytical expressions for $H_{\lambda}\left(\tilde{Y}_{[R S S]}\right), H_{\lambda}\left(\tilde{X}_{R S S}\right)$ and $H_{\lambda}\left(\tilde{X}_{R S S}, \tilde{Y}_{[R S S]}\right)$ in the copula model of FGM family is obtained as

where

$$
\begin{aligned}
& H_{\lambda}\left(\tilde{Y}_{[R S S]}\right)=w_{\alpha, \lambda}(n)=w_{-\alpha, \lambda}(n)=\sum_{r=1}^{n} J_{\alpha, \lambda}(r, n), \\
& H_{\lambda}\left(\tilde{X}_{R S S}, \tilde{Y}_{[R S S]}\right)=H_{\lambda}\left(\tilde{X}_{R S S}\right)+\frac{n}{\lambda-1} \log [2 \alpha(\lambda+1)(2 x-1)],
\end{aligned}
$$

$$
J_{\alpha, \lambda}(r, n)=\frac{1}{1-\lambda} \log \left[\frac{\left(1+\alpha\left(\frac{n-2 r+1}{n+1}\right)\right)^{\lambda+1}-\left(1-\alpha\left(\frac{n-2 r+1}{n+1}\right)\right)^{\lambda+1}}{2 \alpha\left(\frac{n-2 r+1}{n+1}\right)(\lambda+1)}\right],
$$

and

$$
H_{\lambda}\left(\tilde{X}_{R S S}\right)=\sum_{r=1}^{n} H_{\lambda}\left(V_{(r) r}\right)
$$


In the following lemma, we compare $H_{\lambda}\left(Y_{S R S}\right)$ with $H_{\lambda}\left(Y_{[R S S]}\right)$. This result is stated for $0<$ $\lambda<1$.

Lemma 3.2. $14 H_{\lambda}\left(Y_{[R S S]}\right) \leq H_{\lambda}\left(Y_{S R S}\right)$ for all set sizen $\in \mathbb{N}$ and the equality holds when $n=$ 1 or $\alpha=0$.

Proof. For any $0<\lambda<1$ and all $n \in \mathbb{N}$, we have

$$
\begin{aligned}
H_{\lambda}\left(Y_{[R S S]}\right) & =\sum_{r=1}^{n} H_{\lambda}\left(Y_{[r] r}\right)=\sum_{r=1}^{n} \frac{1}{1-\lambda} \log \int\left[g_{[r] r}(y)\right]^{\lambda} d y \\
& \leq \frac{n}{1-\lambda} \log \sum_{r=1}^{n} \frac{1}{n} \int\left[g_{[r] r}(y)\right]^{\lambda} d y \\
& \leq \frac{n}{1-\lambda} \log \int\left[\frac{1}{n} \sum_{r=1}^{n} g_{[r] r}(y)\right]^{\lambda} d y \\
& =\frac{n}{1-\lambda} \log \int\left[f_{Y}(y)\right]^{\lambda} d y=H_{\lambda}\left(Y_{S R S}\right) .
\end{aligned}
$$

\section{Mutual information of RSS in FGM family}

The mutual information for a bivariate random variables $(X, Y)$ with joint pdf $f_{X, Y}(x, y)$ and marginal pdf's $f_{Y}(y)$ and $f_{X}(x)$ is defined as

$$
M(X, Y)=H(X)+H(Y)-H(X, Y)=H(Y)-H(Y \mid X),
$$

where $H(X, Y)$ is the entropy of $(X, Y)$ and $H(Y \mid X)$ is the conditional entropy of $Y$ given $X$ which is

$$
H(Y \mid X)=-\int_{S} f_{X, Y}(x, y) \log \left(\frac{f_{X, Y}(x, y)}{f_{X}(x)}\right) d x d y
$$

where $S$ is the region $f_{X, Y}(x, y)>0$.

The mutual information is a general measure of statistical dependence between two random variables (see Cover and Thomas, 1991). It captures the extent of any kind of functional dependency. We know that $M(X, Y) \geq 0$, and equality holds if and only if $X$ and $Y$ are statistically independent. Moreover, it has the invariance property under one-to-one transformation of $(X, Y)$.

Lemma 4.1. 15Let $\left(X_{R S S}, Y_{[R S S]}\right)=\left\{\left(X_{(r) r}, Y_{[r] r}\right), r=1,2,3, \cdots, n\right\}$ be a ranked set sample of size $n$ from FGM family, then

$$
M_{\alpha}\left(X_{R S S}, Y_{[R S S]}\right)=H\left(\tilde{Y}_{[R S S]}\right)-H\left(\tilde{X}_{S R S}, \tilde{Y}_{S R S}\right)=M_{\alpha}\left(\tilde{X}_{R S S}, \tilde{Y}_{[R S S]}\right) .
$$


Proof. By using (2.10), (2.11) and (4.2), we have

$$
\begin{aligned}
M_{\alpha}\left(X_{R S S}, Y_{[R S S]}\right) & =H\left(X_{R S S}\right)+H\left(Y_{[R S S]}\right)-H\left(X_{R S S}, Y_{[R S S]}\right) \\
& =H\left(Y_{[R S S]}\right)-H\left(Y_{S R S}\right)-H\left(\tilde{X}_{S R S}, \tilde{Y}_{S R S}\right) \\
& =H\left(\tilde{Y}_{[R S S]}\right)-H\left(\tilde{X}_{S R S}, \tilde{Y}_{S R S}\right) .
\end{aligned}
$$

Now by using (2.13), we have

$$
H\left(\tilde{X}_{S R S}, \tilde{Y}_{S R S}\right)=H\left(\tilde{X}_{R S S}, \tilde{Y}_{[R S S]}\right)-H\left(\tilde{X}_{R S S}\right)
$$

With putting (4.5) in (4.4) the invariance property of mutual information is obvious and proof is complete.

Under the perfect ranking assumption, it is easy to see that

$$
\begin{aligned}
& M_{\alpha}\left(X_{R S S}, Y_{[R S S]}\right)=H\left(\tilde{Y}_{[R S S]}\right)-H\left(\tilde{X}_{S R S}, \tilde{Y}_{S R S}\right) \\
& =\sum_{r=1}^{n} I_{\alpha, n}(r)+\sum_{r=1}^{n} \iint \log \left[1+\alpha\left(2 F_{X}(x)-1\right)\right. \\
& \left.\times\left(2 F_{Y}(y)-1\right)\right] f_{(r) r,[r] r}(x, y) d x d y \\
& =w_{\alpha}(n)+n \int_{0}^{1} \int_{0}^{1} \log [1+\alpha(2 u-1)(2 v-1)] \\
& \times[1+\alpha(2 u-1)(2 v-1)] d u d v \\
& =\sum_{r=1}^{n} M_{\alpha}\left(X_{(r) r}, Y_{[r] r}\right) \text {. }
\end{aligned}
$$

Also, the Pearson correlation coefficient between $\widetilde{X}_{R S S}$ and $\tilde{Y}_{[R S S]}$ in the copula model of FGM family is given by

$$
\rho_{\alpha}\left(\tilde{X}_{R S S}, \tilde{Y}_{[R S S]}\right)=\sum_{r=1}^{n} \rho_{\alpha}\left(X_{(r) r}, Y_{[r] r}\right),
$$

where an explicit expression for $\rho_{\alpha}\left(X_{(r) r}, Y_{[r] r}\right)$ is obtained by Tahmsebi and Behboodian(2012) as

$$
\rho_{\alpha}\left(X_{(r) r}, Y_{[r] r}\right)=2 \alpha \sqrt{\frac{r(n-r+1)}{3(n+1)^{2}(n+2)-\alpha^{2}(n-2 r+1)^{2}(n+2)}} .
$$

Table 2. $M_{\alpha}\left(\tilde{X}_{R S S}, \tilde{Y}_{[R S S]}\right)$ and $\rho_{\alpha}\left(\tilde{X}_{R S S}, \tilde{Y}_{[R S S]}\right)$ for the copula model of FGM family.

\begin{tabular}{|c|c|cccc|cccc|}
\hline & & \multicolumn{5}{|c|}{$M_{\alpha}\left(\tilde{X}_{R S S}, \tilde{Y}_{[R S S]}\right)$} & \multicolumn{4}{c|}{$\rho_{\alpha}\left(\tilde{X}_{R S S}, \tilde{Y}_{[R S S]}\right)$} \\
\hline$\alpha$ & & 0.25 & 0.5 & 0.75 & 1 & 0.25 & 0.5 & 0.75 & 1 \\
\hline$n$ & 1 & 0.0034 & 0.0141 & 0.0324 & 0.0599 & 0.0833 & 0.1666 & 0.2500 & 0.3330 \\
& 2 & 0.0046 & 0.0188 & 0.0438 & 0.0824 & 0.1362 & 0.2734 & 0.4124 & 0.5546 \\
& 3 & 0.0050 & 0.0212 & 0.0496 & 0.0942 & 0.1765 & 0.3548 & 0.5370 & 0.7251 \\
& 4 & 0.0054 & 0.0226 & 0.0530 & 0.1014 & 0.2100 & 0.4226 & 0.6404 & 0.8668 \\
& 5 & 0.0056 & 0.0234 & 0.0554 & 0.1065 & 0.2389 & 0.4813 & 0.7302 & 0.9898 \\
\hline
\end{tabular}

Table 2 provides the values of $M_{\alpha}\left(X_{R S S}, Y_{[R S S]}\right)$ and $\rho_{\alpha}\left(X_{R S S}, Y_{[R S S]}\right)$ as a function of $n$ and $\alpha$ for $n=1(1) 5$, and $\alpha=0.25(0.25) 1$. These values are derived by using (4.6) and (4.8). As Table 2 and easy computations show, $M_{\alpha}\left(X_{R S S}, Y_{[R S S]}\right)$ and $\rho_{\alpha}\left(X_{R S S}, Y_{[R S S]}\right)$ for the copula model of FGM family have the following properties 
(i) $\left|M_{\alpha}\left(X_{R S S}, Y_{[R S S]}\right)\right|<\rho_{\alpha}\left(X_{R S S}, Y_{[R S S]}\right)$,

(ii) $\rho_{\alpha}\left(X_{R S S}, Y_{[R S S]}\right)=-\rho_{-\alpha}\left(X_{R S S}, Y_{[R S S]}\right)$,

(iii) for fixed $n, M_{\alpha}\left(X_{R S S}, Y_{[R S S]}\right)$ and $\rho_{\alpha}\left(X_{R S S}, Y_{[R S S]}\right)$ increase as $|\alpha|$ increase,

(iv) for fixed $\alpha, M_{\alpha}\left(X_{R S S}, Y_{[R S S]}\right)$ and $\rho_{\alpha}\left(X_{R S S}, Y_{[R S S]}\right)$ increase as $n$ increase,

(v) $M_{\alpha}\left(X_{R S S}, Y_{[R S S]}\right) \leq .05 n$.

\section{Kullback-Leibler information of RSS in FGM family}

The Kullback-Leibler (KL) discrimination information between two continuous random variables $Z_{1}$ and $Z_{2}$ with pdf's $f_{1}$ and $f_{2}$, respectively, is given by

$$
K L\left(Z_{1}, Z_{2}\right)=\int_{-\infty}^{+\infty} f_{1}(z) \log \left(\frac{f_{1}(z)}{f_{2}(z)}\right) d z=E_{1}\left(\log \frac{f_{1}(Z)}{f_{2}(Z)}\right),
$$

where $E_{1}$ denotes the expectation with respect to $f_{1} . K\left(Z_{1}, Z_{2}\right)$ is non-negative and it is not symmetric.

The KL discrimination information between $X_{R S S}$ and $X_{S R S}$ is distribution-free, and is defined by Jafari Jozani. and Ahmadi (2014) as

$$
K L\left(X_{R S S}, X_{S R S}\right)=-\sum_{r=1}^{n} \log r\left(\begin{array}{l}
n \\
r
\end{array}\right)+n(n-1):=d_{n} .
$$

Note that $\left\{d_{n}, n=1,2, \ldots\right\}$ is a nondecreasing sequence of non-negative real values for all $n \in \mathbb{N} . K L\left(X_{R S S}, X_{S R S}\right)$ increases as $n$ increases. Also, the KL discrimination information between $X_{S R S}$ and $X_{R S S}$ is distribution-free, and is given by

$$
K L\left(X_{S R S}, X_{R S S}\right)=-k(n) .
$$

In the following lemma, we compare $K L\left(X_{S R S}, Y_{S R S}\right)$ with $K L\left(X_{R S S}, Y_{[R S S]}\right)$ in FGM family.

Lemma 5.1. 16Let $\left(X_{R S S}, Y_{[R S S]}\right)=\left\{\left(X_{(r) r}, Y_{[r] r}\right), r=1,2,3, \cdots, n\right\}$ be a ranked set sample of size $n$ fromFGM family, then

$$
K L\left(X_{S R S}, Y_{S R S}\right) \leq K L\left(X_{R S S}, Y_{[R S S]}\right)
$$


Proof. To show the result note that

$$
\begin{aligned}
K L\left(X_{R S S}, Y_{[R S S]}\right)= & \sum_{r=1}^{n} \int f_{(r) r}(x) \log \left\{\frac{f_{(r) r}(x)}{g_{[r] r}(y)}\right\} d x \\
= & \sum_{r=1}^{n} K L\left(X_{(r) r}, Y_{[r] r}\right) \\
= & \sum_{r=1}^{n} \int f_{(r) r}(x) \log f_{(r) r}(x) d x-\sum_{r=1}^{n} \log g_{[r] r}(y) \\
= & -H\left(X_{R S S}\right)-\sum_{r=1}^{n} \log g_{[r] r}(y) \\
& \geq-H\left(X_{S R S}\right)-n \log f_{Y}(y) \int f_{X}(x) d x \\
= & n \int f_{X}(x) \log \left[\frac{f_{X}(x)}{f_{Y}(y)}\right] d x=K L\left(X_{S R S}, Y_{S R S}\right),
\end{aligned}
$$

which complete the proof.

Under the perfect ranking assumption, the KL discrimination information between $Y_{S R S}$ and $Y_{[R S S]}$ is distribution-free, and is obtained

$$
\begin{aligned}
K L\left(Y_{S R S}, Y_{[R S S]}\right) & =\sum_{r=1}^{n} \int f_{Y}(y) \log \left[\frac{f_{Y}(y)}{g_{[r] r}(y)}\right] d y \\
& =\sum_{r=1}^{n} K\left(Y, Y_{[r] r}\right) \\
& =-\sum_{r=1}^{n} \int f_{Y}(y) \log \left[1+\frac{n-2 r+1}{n+1} \alpha\left(1-2 F_{Y}(y)\right)\right] d y \\
& =-\sum_{r=1}^{n} \int_{0}^{1} \log \left[1+\frac{n-2 r+1}{n+1} \alpha(1-2 u)\right] d u \\
& =-\sum_{r=1}^{n} t_{\alpha, n}(r)=z_{\alpha}(n),
\end{aligned}
$$

where

$$
\begin{aligned}
t_{\alpha, n}(r)= & \frac{n+1}{2 \alpha(n-2 r+1)}\left\{\left(1-\left(\frac{n-2 r+1}{n+1}\right) \alpha\right)\left[\log \left(1-\left(\frac{n-2 r+1}{n+1}\right) \alpha\right)-1\right]\right. \\
& \left.-\left(1+\left(\frac{n-2 r+1}{n+1}\right) \alpha\right)\left[\log \left(1+\left(\frac{n-2 r+1}{n+1}\right) \alpha\right)-1\right]\right\} .
\end{aligned}
$$

Furthermore the KL divergence for $Y_{[R S S]}$ and $Y_{S R S}$ is distribution-free, and is given by 


$$
\begin{aligned}
K L\left(Y_{[R S S]}, Y_{S R S}\right) & =\sum_{r=1}^{n} \int g_{[r] r}(y) \log \left\{\frac{g_{[r] r}(y)}{f_{Y}(y)}\right\} d y \\
& =\sum_{r=1}^{n} K\left(Y_{[r] r}, Y\right) \\
& =-\sum_{r=1}^{n} \int g_{[r] r}(y) \log \left[1+\frac{n-2 r+1}{n+1} \alpha\left(1-2 F_{Y}(y)\right)\right] d y \\
& =-\sum_{r=1}^{n} \int_{0}^{1}\left[1+\frac{n-2 r+1}{n+1} \alpha(1-2 u)\right] \log \left[1+\frac{n-2 r+1}{n+1} \alpha(1-2 u)\right] d u \\
& =-\sum_{r=1}^{n} I_{\alpha, n}(r)=-w_{\alpha}(n)=-H\left(\tilde{Y}_{[R S S]}\right) .
\end{aligned}
$$

We known that the KL divergence is non-symmetric and can not be considered as a distance metric. So, the following symmetric Kullback-Leibler (SKL) distance between $Y_{S R S}$ and $Y_{[R S S]}$ is given by

$$
S K L\left(Y_{S R S}, Y_{[R S S]}\right)=K L\left(Y_{S R S}, Y_{[R S S]}\right)+K L\left(Y_{[R S S]}, Y_{S R S}\right)=z_{\alpha}(n)-w_{\alpha}(n) .
$$

The Rényi information of order $\lambda$ between $Z_{1}$ and $Z_{2}$ with marginal pdf's $f_{1}$ and $f_{2}$ is defined as

$$
K_{\lambda}\left(Z_{1}, Z_{2}\right)=\frac{1}{\lambda-1} \log \int\left[\frac{f_{1}(z)}{f_{2}(z)}\right]^{\lambda-1} f_{1}(z) d z
$$

where $K(X, Y)=\lim _{\lambda \rightarrow 1} K_{\lambda}(X, Y)$ is the $\mathrm{KL}$ information between $X$ and $Y$. The Rényi information of order $\lambda$ between $X_{R S S}$ and $X_{S R S}$ is distribution-free, and is derived by

$$
K_{\lambda}\left(X_{R S S}, X_{S R S}\right)=\sum_{r=1}^{n} \frac{1}{\lambda-1} \log \int\left[\frac{f_{(r) r}(x)}{f(x)}\right]^{\lambda-1} f(x) d x=-\sum_{r=1}^{n} H_{\lambda}\left(V_{(r) r}\right) .
$$

Also, the Rényi information of order $\lambda$ between $Y_{[R S S]}$ and $Y_{S R S}$ is distribution-free, and is obtained by

$$
K_{\lambda}\left(Y_{[R S S]}, Y_{S R S}\right)=\sum_{r=1}^{n} \frac{1}{\lambda-1} \log \int\left[\frac{g_{([r] r}(y)}{f(y)}\right]^{\lambda-1} f(y) d x=-\sum_{r=1}^{n} J_{\alpha, \lambda}(r, n) .
$$

\section{Acknowledgements}

The authors would like to thank the Editor and the Referee for their valuable comments and suggestions that resulted in improving the quality of presentation of this manuscript. 


\section{References}

[1] Abbasnejad, M. and Arghami, N. R. (2011). Rényi entropy properties of records. Journal of Statistical Planning and Inference, 141(7), 2312-2320.

[1] Abo-Eleneen, Z. A. (2001). Information in Order Statistics and Their Concomitants and Applications. Phd. Dissertation, Zagazig University, Egypt.

[2] Abo-Eleneen, Z. A. (2011). The entropy of progressively censored samples. Entropy, 13(2), 437-449.

[3] Abo-Eleneen, Z. A. and Nagaraja, H. N. (2002). Fisher information in an order statistic and its concomitant. Annals of the Institute of Statistical Mathematics, 54(3), 667-680.

[4] Ahmadi, J. and Fashandi, M. (2008). Shannon information properties of the endpoints of record coverage. Communications in Statistics - Theory and Methods, 37(4), 481-493.

[5] Balakrishnan, N., Habibi Rad, A., and Arghami, N. R. (2007). Testing exponentiality based on Kullback-Leibler information with progressively Type-ii censored data. IEEE Transactions on Reliability, 56(2), 301-307.

[6] Barabesi, L. and El-Sharaawi, A. (2001). The efficiency of ranked set sampling for parameter estimation. Statistics \& Probability Letters, 53(2), 189-199.

[7] Baratpour, S., Ahmadi, J., and Arghami, N. R. (2007a). Entropy properties of record statistics. Statistical Papers, 48(2), 197-213.

[8] Baratpour, S., Ahmadi, J., and Arghami, N. R. (2007b). Some characterizations based on entropy of order statistics and record values. Communications in Statistics - Theory and Methods, 36(1), 47-57.

[9] Baratpour, S., Ahmadi, J., and Arghami, N. R. (2008). Characterizations based on Rényi entropy of order statistics and record values. Journal of Statistical Planning and Inference, 138(8), 2544-2551.

[10] Chen, Z. (2000). The efficiency of ranked-set sampling relative to simple random sampling under multi-parameter families. Statistica Sinica, 10(1), 247-264.

[11] Cover, T. M. and Thomas, J. A. (1991). Elements of information theory. John Wiley \& Sons, New York.

[12] Ebrahimi, N., Soofi, E. S., and Zahedi, H. (2004). Information properties of order statistics and spacings. Information Theory, IEEE Transactions on, 50(1), 177-183. 
[13] Fashandi, M. and Ahmadi, J. (2012). Characterizations of symmetric distributions based on Rényi entropy. Statistics \& Probability Letters, 82(4), 798-804.

[14] Frey, J. (2013). A note on Fisher information and imperfect ranked-set sampling. Communications in Statistics - Theory and Methods, 10.1080/03610926.2012.683131.

[15] Habibi Rad, A., Yousefzadeh, F., and Balakrishnan, N. (2011). Goodness-of-fit test based on kullback-leibler information for progressively type-ii censored data. Reliability, IEEE Transactions on, 60(3), 570-579.

[16] Harris, B. (1982). Entropy, S. Kotz, N.L. Johnson, Encyclopedia of Statistical Sciences, volume 2, pages 512-516. Wiley.

[17] Jafari Jozani, M. and Ahmadi, J. (2014). On uncertainty and information properties of ranked set samples. Information Sciences 264, 291-301.

[18] Madadi, M. and Tata, M. (2011). Shannon information in record data. Metrika, 74(1), 11-31.

[19] McIntyre, G. A. (1952). A method for unbiased selective sampling, using ranked sets. Australian Journal of Agricultural Research, 3(4), 385-390.

[20] Meyer, C. (2009). The bivariate normal copula. http://arxiv.org/pdf/0912.2816.pdf.

[21] Nayak, I. K. (1985). On diversity measures based on entropy functions. Communications in Statistics - Theory and Methods, 14(1), 203-215.

[22] Nelson, R. B. (1999). An Introduction to Copulas. Springer, New York.

[23] Park, S. (1995). The entropy of consecutive order statistics. IEEE Transactions on Information Theory, 41(6), 2003-2007.

[24] Park, S. (2005). Testing exponentiality based on the Kullback-Leibler information with the type ii censored data. IEEE Transactions on Reliability, 54(1), 22-26.

[25] Park, S. and Lim, J. (2012). On the effect of imperfect ranking on the amount of Fisher information in ranked set samples. Communications in Statistics - Theory and Methods, 41(19), 3608-3620.

[26] Raqab, M. Z. and Awad, A. M. (2000). Characterizations of the Pareto and related distributions. Metrika, 52(1), 63-67.

[27] Raqab, M. Z. and Awad, A. M. (2001). A note on characterization based on shannon entropy of record statistics. Statistics, 35(4), 411-413. 
[28] Scaria, J. and Nair, N. U. (1999). On concomitants of order statistics from Morgenstern family. Biometrical Journal, 41(4), 483-489.

[29] Shannon, C. (1948). A mathematical theory of communication. Bell System Technical Journal, 27, 379-432.

[30] Soofi, E. S. (2000). Principal information theoretic approaches. Journal of the American Statistical Association, 95(452), 1349-1353.

[31] Stokes, S. L. (1977). Ranked set sampling with concomitant variables. Communications in Statistics - Theory and Methods, 6(12), 1207-1211.

[32] Tahmasebi, S. and Behboodian, J. (2012). Information properties for concomitants of order statistics in Farlie-Gumbel-Morgenstern (FGM) family. Communications in Statistics Theory and Methods, 41(11), 1954-1968.

[33] Wong, K. M. and Chen, S. (1990). The entropy of ordered sequences and order statistics. IEEE Transactions on Information Theory, 36(2), 276-284.

[34] Zahedi, H. and Shakil, M. (2006). Properties of entropies of record values in reliability and life testing context. Communications in Statistics - Theory and Methods, 35(6), 997-1010.

Received December 12, 2013; accepted July 26, 2014.

Saeid Tahmasebi

Department of Statistic

Persian Gulf University

Bushehr, Iran

tahmasebi@pgu.ac.ir

Ali Akbar Jafari

Department of Statistics

Yazd University, Yazd, Iran

aajafari@yazd.ac.ir 